\title{
On The Enumeration of The Transitive and Acyclic Digraphs Having a Fixed Support Set
}

\author{
Khalid Shea Khairalla Al'Dzhabri \\ University of Al-Qadisiyah, College of Education, Department of mathematics \\ Khalid.aljabrimath@qu.edu.iq
}

Recived : $18 \backslash 10 \backslash 2017$

Revised : 30\11\2017

Accepted : $6 \backslash 12 \backslash 2017$

Available online : $\quad 20 / 1 / 2018$

DOI: 10.29304/jqcm.2018.10.1.336

\begin{abstract}
In previous works of the author, the concept of a binary reflexive adjacency relations was introduced on the set of all binary relations of the set $X$, and an algebraic system consisting of all binary relations of the set $X$ and of all unordered pairs of adjacent binary relations was defined. If $X$ is a finite set, then this algebraic system is a graph (graph of binary relations $G$ ). The current paper introduces the notion of a support set for acyclic and transitive digraphs. This is the collections $S(\sigma)$ and $S^{\prime}(\sigma)$ consisting of the vertices of the digraph $\sigma \in G$ that have zero indegree and zero outdegree, respectively. It is proved that if $G_{\sigma}$ is a connected component of the graph $G$ containing the acyclic or transitive digraph $\sigma \in G$, then $\left\{S(\tau): \tau \in G_{\sigma}\right\}=\left\{S^{\prime}(\tau): \tau \in G_{\sigma}\right\}$. A formula for the number of acyclic and transitive digraphs having a fixed support set is obtained.
\end{abstract}

Keywords: Enumeration of graphs, acyclic digraph, transitive digraph. Mathematics subject classification :05C30 


\section{Definitions and auxiliary propositions:}

Definition 1.1 Any "binary relation $\sigma \subseteq X^{2}(X-$ arabitrary set), generates a

charcteristic function" $\sigma^{\prime}: X^{2} \rightarrow\{0,1\}$, (if $(x, y) \in \sigma$, then $\sigma^{\prime}(x, y)=1, \quad$ otherwise $\left.\sigma^{\prime}(x, y)=0\right)$, and this mpping is bijectve.

Remarks 1.2 1) From the definition(1.1) we called the subset $\sigma \subseteq X^{2}$ as the relationsships and functions (someetimes digraphs).

2) If $X$ finite set then the characeteristic function can be interepreted as a binaary matrix (the matrix consisiting of 0 and 1). Definition 1.3 The relations $\sigma, \tau \subseteq X^{2}$ called adjaceent if there exists a disjoint of union of two subsets $X=Y \cup Z$, such that:

i) $\sigma(x, y)=0$ for all $(x, y) \in Y \times Z$;

ii) $\tau(x, y)=0$ for all $(x, y) \in Z \times Y$;

iii) $\quad \tau(x, y)+\sigma(y, x)=1 \quad$ for $\quad$ all $(x, y) \in Y \times Z$

iv) $\quad \sigma(x, y)=\tau(x, y)$ for all $(x, y) \in Y^{2} \cup Z^{2}$.

Remark 1.4 From the definiition (1.3), that if the relation $\tau$ adjaceent with a relation $\sigma$, then $\sigma$ adjaceent with a relation $\tau$, and this fact we write in the form of a diagaram

\section{$\sigma \stackrel{Y \times Z}{\longleftrightarrow} \tau$.}

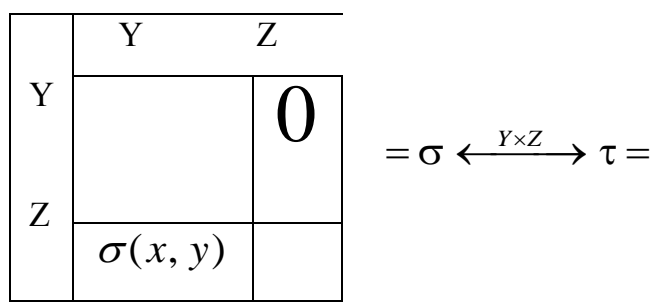

Example 1.5: For $X=\{1,2,3,4\}$ we have the following adjaceent relations:

$\left[\begin{array}{cccc}1 & 0 & 0 & 0 \\ 0 & 1 & 0 & 0 \\ 1 & 1 & 1 & 1 \\ 1 & 1 & 0 & 1\end{array}\right] \stackrel{\{1\} \times\{2,3,4\}}{\stackrel{(}{\longrightarrow}}\left[\begin{array}{cccc}1 & 1 & 0 & 0 \\ 0 & 1 & 0 & 0 \\ 0 & 1 & 1 & 1 \\ 0 & 1 & 0 & 1\end{array}\right]$


Khalid .S

\section{Example1.6:}

$$
X=\{1, \ldots, 6\}, Y=\{1,2\}, Z=\{3,4,5,6\} \text {, }
$$

then the adjaceent relation is:

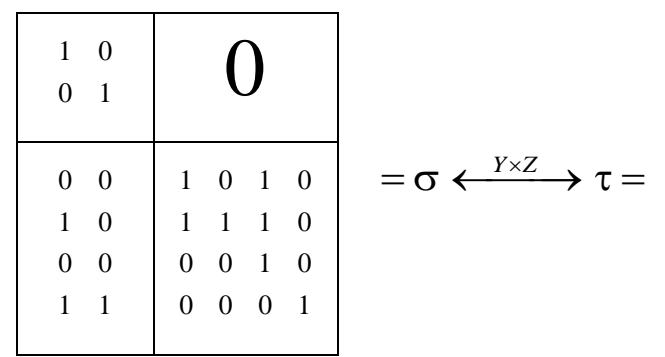

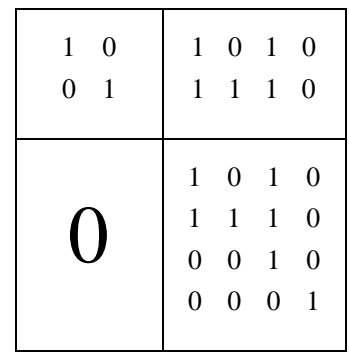

"Thus, the set $X$ generatees a pair $\left\langle 2^{X^{2}}, E(X)\right\rangle$, where $2^{X^{2}}$ is the set of vertices, consist of the set of all binary relations of the set $X$, and $E(X)-$ is a set of edeges, consist of all unordered distinct pairs of adjaceent of binary relations of the set $X$. The pair $G(X) \doteq\left\langle 2^{X^{2}}, E(X)\right\rangle$ will be called "undirected graph of binary relations of the set $X$ \%

The following theorem proved that in our work [3].

Theorem 1.7: If $\operatorname{card} X \neq 1$, then $\operatorname{diam}(G(X))=2$.

Remark 1.8: we denoted the "connected component of the graph" $G(X)$ by $G_{\sigma}(X)$, which contaiins the given relation $\sigma \in 2^{X^{2}}$.

\section{Arithmetic properties of some subgraphs of the} graph of binary relations. "We denoted that the collection of all partial orders defined on the set $X$ by $V_{0}(X)$. And the collection of all reflexivie transitive relations defined on the set $X$ by $V(X)$ and where $X$ finiite sets the collection of all acyclic relations by $A(X)$."

In [1],[2] and [3] we proved that if $\sigma, \tau \in 2^{X^{2}}$ are adjaceent then:

1. $\sigma \in V_{0}(X)$ if and only if $\tau \in V_{0}(X)$;

2. $\sigma \in V(X)$ if and only if $\tau \in V(X)$;

3. $\sigma \in A(X)$ if and only if $\tau \in A(X)$.

Therefore, in the garaph $\left\langle 2^{X^{2}}, E(X)\right\rangle$ define the following subgrphs:

$$
\left\langle V_{0}(X), E(X)\right\rangle, \quad\langle V(X), E(X)\rangle, \quad\langle A(X), E(X)\rangle_{\ldots}
$$

Continue to suggeset that card $X<\infty$ (i.e $X=\{1, \ldots, n\})$. Then we get the following remakes:

Remakes 2.1: 1) If replacing the unit elements $\sigma(x, x)$, zeros, then we get a one-to-one corresepondence between the set $V_{0}(X)$ and the set of all labeled transitive digraphs denoted by $V_{0}^{0}(X)$

2) There exist a one-to-one corresepondence between the set $V_{0}(X)$ and the seet of all labeeled $T_{0}$-topoology denoted by $\mathrm{T}_{0}(X)$. 
3) Let

$$
T_{0}(n)=\operatorname{card} \mathrm{T}_{0}(X)=\operatorname{card} V_{0}(X)=\operatorname{card} V_{0}^{0}(X) \text {. }
$$

Additional suggest that $T_{0}(0)=1$.

In [1] we proved that the number of "connected component of the graph " $\left\langle V_{0}(X), E(X)\right\rangle$ equal to $T_{0}(n-1)$. We note that for any nataural number $n$ the following equaliities are hold:

$$
T_{0}(n)=\sum_{p_{1}+\ldots+\mathrm{p}_{k}=n} \frac{n !}{p_{1} !+\ldots+\mathrm{p}_{k} !} V\left(p_{1}, \ldots, \mathrm{p}_{k}\right)
$$

$T_{0}(n)=\sum_{p_{1}+\ldots+\mathrm{p}_{k}=n}(-1)^{n-k} \frac{n !}{p_{1} !+\ldots+\mathrm{p}_{k} !} w\left(p_{1}, \ldots, \mathrm{p}_{k}\right), \operatorname{card} A(X)=\sum_{p_{1}+\ldots+p_{k}=n}(-1)^{n-k} \frac{n !}{p_{1} ! \ldots p_{k} !} 2^{\left(n^{2}-p_{1}^{2}-\ldots-p_{k}^{2}\right) / 2}, \ldots$ where the summation is over all ordered sets $\left(p_{1}, \ldots, p_{k}\right)$ of positivee integers such that $p_{1}+\ldots+p_{2}=n$. The first formula see [4],[5] and [6] and second in [7]. The number of $V\left(p_{1}, \ldots, p_{k}\right)$ and $W\left(p_{1}, \ldots, p_{k}\right)$ denote the number of partial orders of a special form, which depends on a set of $\left(p_{1}, \ldots, p_{k}\right)$ see below (4).

4) If $X=\{1, \ldots, n\}$. Then:

$$
\operatorname{cardV}(X)=\sum_{m=1}^{n} S(n, m) T_{0}(m) \text { see [4],[8] }
$$

and [9] where $S(n, m)-$ This Stiriling numbers of the 2 nd kind in our work [2] we proveed that the number of coonnected component of the graph $\langle V(X), E(X)\rangle \quad$ equal

to

$\sum_{m=1}^{n} S(n, m) T_{0}(m-1)$

Remark 2.2 From above there exists a one to one correseponded between the set of all transitivereflexive relations $V(X)$ and the set of all labeled topologies $T(X)$ defined on the set $X$. If $A_{n}=A(X)$ and $X=\{1, \ldots, n\}$ a cording to [10] the following equality holds:

In our work [3] we proveed that the number of connected component of the graph $\langle A(X), E(X)\rangle \quad$ equal to

$$
\sum_{p_{1}+\ldots+p_{k}=n}(-1)^{n-k} \frac{(n-1) !}{\left(p_{1}-1\right) ! p_{2} ! \ldots p_{k} !} 2^{\left(n^{2}-p_{1}^{2}-\ldots-p_{k}^{2}\right) / 2} \ldots . .
$$

In both cases, the summation is over all ordered sets $\left(p_{1}, \ldots, p_{k}\right)$ of natural numbers such that $p_{1}+p_{2}+\ldots+p_{k}=n$.

Remark 2.3 We note that the formulass (2) and (3) have the same struccture, and in the second case if the formula has a finisihed appearance, then in the first formula remains a problem of calculation of numbers $W\left(p_{1}, \ldots, p_{k}\right)$. 
In the work [10] the following (more general) assertions are proved. We denote by $A_{n}(x)=\sum_{r} A_{n r} x^{r}$ a polynomial whose coefficient $A_{n r}$ is equal to the number of labeled acyclic digraphs of order $n$ having exactly $r$ arcs. It is clear that $A_{n}=A_{n}(1)$. We use the agreement $A_{0}(x)=A_{0}=1$. The polynomial $A_{n}(x)$ for $n \in \square$ is given by the formula:

$$
A_{n}(x)=\sum_{p_{1}+\ldots+p_{k}=n}(-1)^{n-k} \frac{n !}{p_{1} ! \ldots p_{k} !}(1+x)^{\left(n^{2}-p_{1}^{2}-\ldots-p_{k}^{2}\right) / 2}
$$

(compare with the formula (3). For any $n \geq 0$, we have the following :

$$
\sum_{m=0}^{n}(-1)^{m}\left(\begin{array}{c}
n \\
m
\end{array}\right)(1+x)^{m(n-m)} A_{m}(x)=\delta_{n 0} .
$$

Now from the formulass above we give the following example:

\section{Examples of subgraphs of the graph of binary} relations.

Let $X=\{1,2,3\}$.Then we get 3 "connected components of the graph" $\left\langle V_{0}(X), E(X)\right\rangle$, contains 19 partial orders:

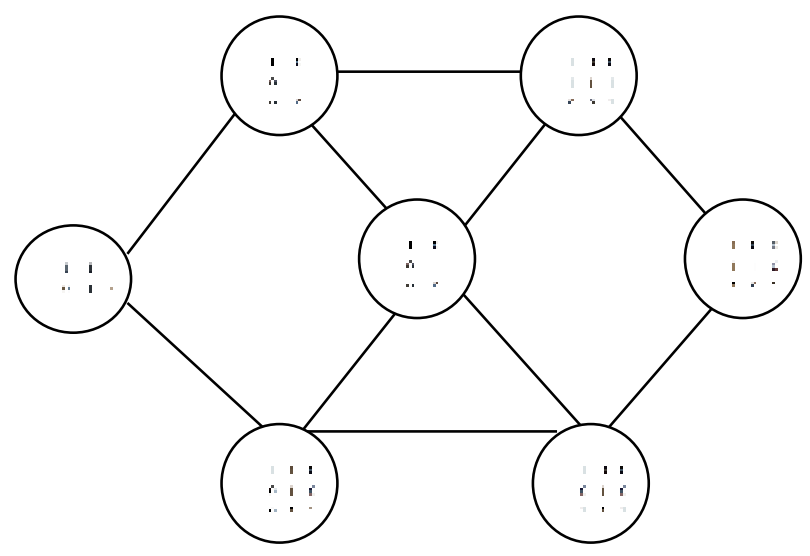

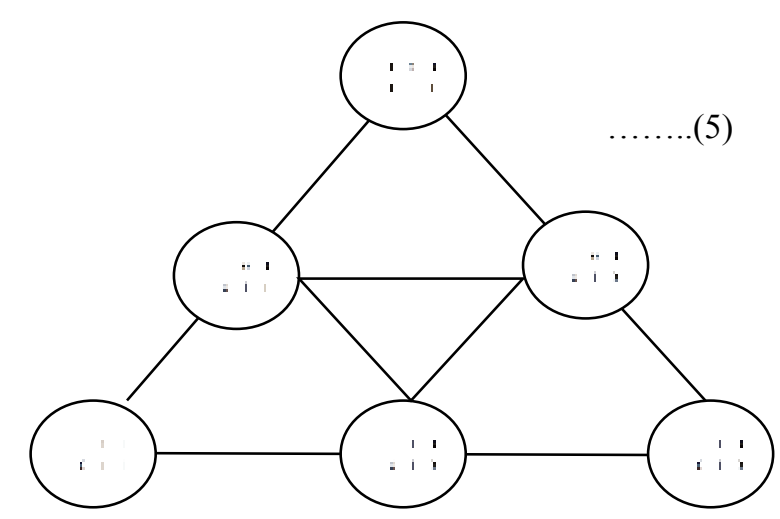

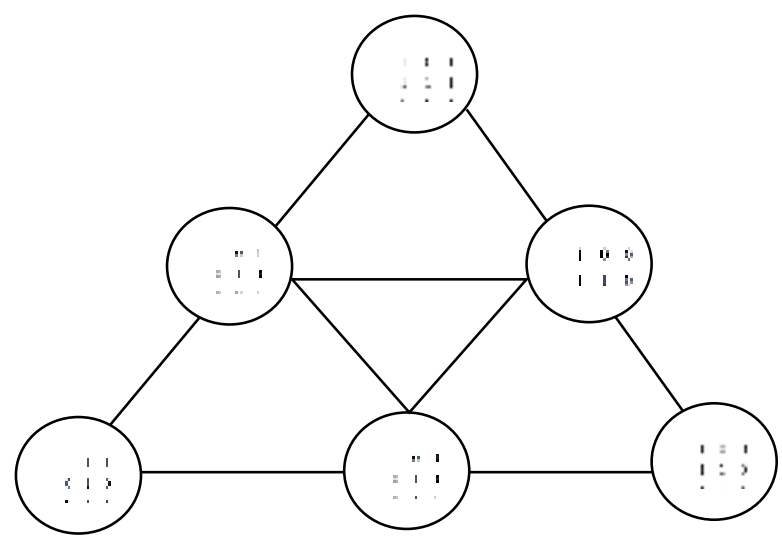

And the graph $\langle V(X), E(X)\rangle$, contains 29 reflexive- transitive relations. It has 7 conneected componeents: 3 components of the graph (4) above and 4 components as:

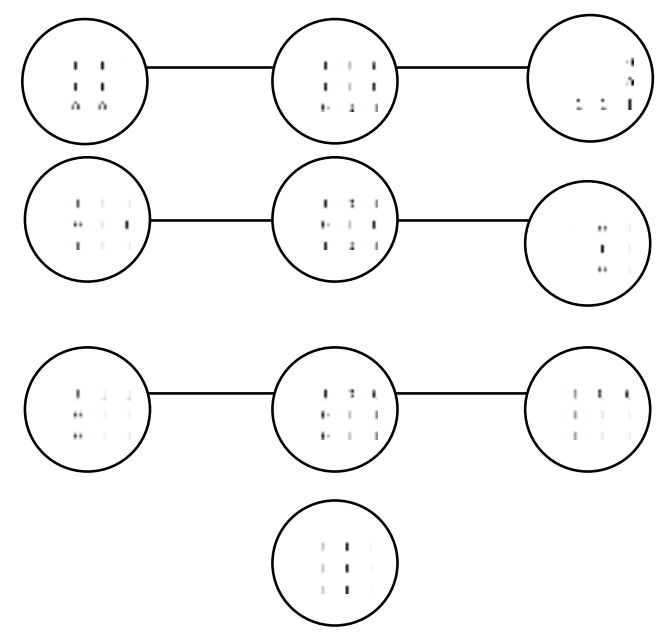


And the graph $\langle A(X), E(X)\rangle$ contaiins 25 acyclice relations. It has 5 connected components : 3 components of the grapha (4) above (in them should be replaced the elementes of the set $V_{0}(X)$ by the elements of the set $\left.V_{0}^{0}(X)\right) 2$ componenets as follows:

"Where $X=\{1,2,3,4\}$ in subgraphs of the form (1) there is a 219,355 and 543 vertices respectively and the number of connected components of these subgrags in (1) equal to 19,45 , and 79 respectively."
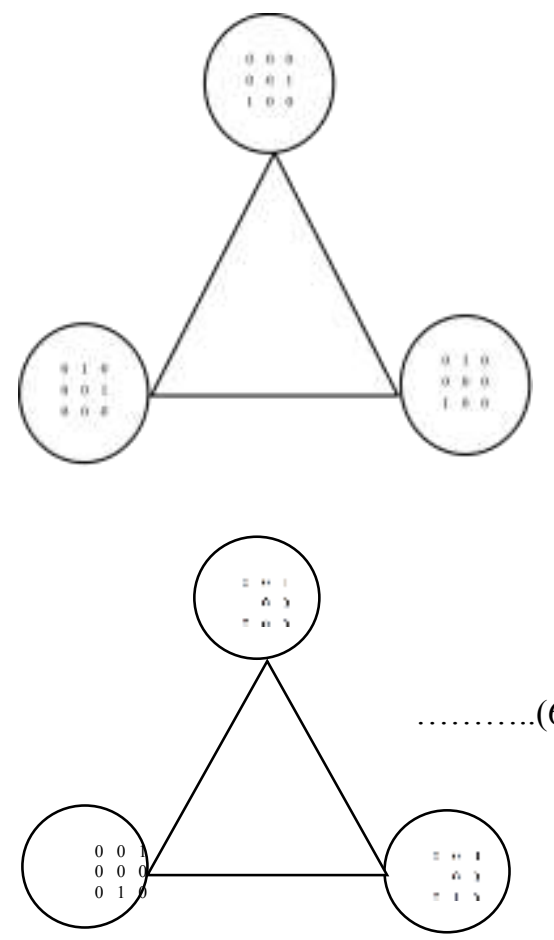

4. Definition of numbers $W\left(p_{1}, \ldots, p_{k}\right)$ and $V\left(p_{1}, \ldots, p_{k}\right):$ We fix $\left(p_{1}, \ldots p_{k}\right) \in \square^{k}$, and let $n=p_{1}+\ldots+p_{k}, X=\{1, \ldots, n\}$. The set $\left(p_{1}, \ldots, p_{k}\right)$ Will be called a partition of the number $n$. The partition $\left(p_{1}, \ldots, p_{k}\right)$ corresponds to representation of a relation $\sigma \in V_{0}(X)$ in block form

$$
\left|\begin{array}{cccc}
\sigma^{11} & \sigma^{12} & \cdots & \sigma^{1 k} \\
\sigma^{21} & \sigma^{22} & \cdots & \sigma^{2 k} \\
\cdots & \cdots & \cdots & \cdots \\
\sigma^{k 1} & \sigma^{k 2} & \cdots & \sigma^{k k}
\end{array}\right|
$$

In which at the intersection of the $i^{\text {th }}$ horizontal and $j^{\text {th }}$ vertical bands cost a block $\sigma^{i j}$ with $p_{i}$ rows and $p_{j}$ columns recording the relation $\sigma \in V_{0}(X)$ in the form (7) will be called a block representation of type $\left(p_{1}, \ldots, p_{k}\right)$. Through $w\left(p_{1}, \ldots, p_{k}\right)$ we denote the set of all relations $\sigma \in V_{0}(X)$ for which in the block representation (7) of type $\left(p_{1}, \ldots, p_{k}\right)$ :

1- $\quad$ All blocks $\sigma^{i j}, 1 \leq j<i \leq k$, consist of zeros;All diagonal blocks $\sigma^{i i}, i=1, \ldots, k,-$ identity matrices. And let $W\left(p_{1}, \ldots, p_{k}\right)=\operatorname{card} w\left(p_{1}, \ldots, p_{k}\right)$.

Through $v\left(p_{1}, \ldots, p_{k}\right)$ we denote the set of all relations $\sigma \in \omega\left(p_{1}, \ldots, p_{k}\right)$ such that in block representation (7) of type $\left(p_{1}, \ldots, p_{k}\right)$ in superdiagonal blocks $\sigma^{i-1, i}, i=2, \ldots, k$ in each column there is at least number one we call such blocks (nondegenerate). We note that, by virtue of transitivity $\sigma \quad$ all blocks $\sigma^{s r}, s=1, \ldots k-1, r=s+1, \ldots, k$ nondegenerate. We use the notation $V\left(p_{1}, \ldots, p_{k}\right)=\operatorname{cardv}\left(p_{1}, \ldots, p_{k}\right)$. 


\section{Comparison of the formulas (2) and (3):} Inclusion $v_{0}^{0}(X) \subset A(X)$ it is well known that formulas (2) and (3) for calculating numbers $\operatorname{cardv} v_{0}^{0}(X)$ and $\operatorname{card} A(X)$ have the same structure. However, if in the second case the formula has a completed form, then in the first the problem persists calculation of numbers $W\left(p_{1}, \ldots, p_{k}\right)$. In the framework of studies of this problem in the works $[7,11]$ obtained family of equations of connection between the individual numbers $W\left(p_{1}, \ldots, p_{k}\right)$ : $W\left(p_{\pi(1)}, \ldots, p_{\pi(k)}\right)=W\left(p_{1}, \ldots, p_{k}\right), \quad \pi \in D_{k}$, Where $D_{k}-$ is the dihedral group, generated by substitutions:

$$
\begin{gathered}
\left(\begin{array}{ccccc}
1 & 2 & \ldots & k-1 & k \\
2 & 3 & \ldots & k & 1
\end{array}\right),\left(\begin{array}{ccccc}
1 & 2 & \ldots & k-1 & k \\
k & k-1 & \ldots & 2 & 1
\end{array}\right), \\
\sum_{q_{1}+\ldots+q_{r}=m}(-1)^{m-r} \frac{m !}{q_{1} ! \ldots q_{r} !} W\left(p+1, q_{1}, \ldots, q_{r}\right)= \\
\sum_{q_{1}+\ldots+q_{r}=m}(-1)^{m-r} \frac{m !}{q_{1} ! \ldots q_{r} !}(r+1) W\left(p+1, q_{1}, \ldots, q_{r}\right)
\end{gathered}
$$$$
W(p, 1, q, 1)=\sum_{r=0}^{q}\left(\begin{array}{c}
q \\
r
\end{array}\right)\left(2^{q-r}+1\right)^{p} W(p, r, 1)
$$$$
+\sum_{r=0}^{p}\left(\begin{array}{c}
p \\
r
\end{array}\right)\left(2^{p-r}+1\right)^{q} W(r, q, 1) .
$$

Formulas $\quad W(n)=1, W(p, q)=2^{p q} \quad$ are obvious. B [11], the following explicit formulas are proved:

$$
W(p, 1, q)=\sum_{r=0}^{q}\left(\begin{array}{l}
q \\
r
\end{array}\right)\left(2^{r}+2^{q}\right)^{p},
$$

$$
\begin{aligned}
& W(p, 2, q)=\sum_{q_{1}+\ldots+q_{4}=q} \frac{q !}{q_{1} ! \ldots q_{4} !}\left(2^{q_{1}}+2^{q_{1}+q_{2}}+2^{q_{1}+q_{3}}+2^{q}\right)^{p} \\
& W(p, 1,1, q)-W(p, 2, q)= \\
& =\sum_{q_{1}+q_{2}+q_{3}=q} \frac{q !}{q_{1} ! \cdot q_{2} \cdot q_{3} !}\left(2^{q_{1}}+2^{q_{1}+q_{2}}+2^{q}\right)^{p} .
\end{aligned}
$$

The above relations allow us to calculate the numbers $T_{0}(n)$ for all $n \leq 7$ (without the use of a computer, by solving a system of linear equations with respect to quantities $\left.W\left(p_{1}, \ldots, p_{k}\right)\right)$. In [11] it was noted that to calculate the number $T_{0}(8)$ of these relations is not sufficient (only three equations are

missing with respect to the quantities $W\left(p_{1}, \ldots, p_{k}\right)$ generating the required rank of the matrix of the system). We assume that the existence of some general patterns, generating new relations equation. Note also that due to the development of effective algorithms

Computer calculations [12] currently known value $T_{0}(n)$ for all $n \leq 18$.

\section{The number of transitive and acyclic digraphs} having a fixed support set.

Let $\left(p_{1}, p_{2}\right) \in N^{2}, p=p_{1}, m=p_{2}$,

$X=\{1, \ldots, p+m\}, M=\{p+1, \ldots, p+m\}$. through $w(p ; m)$ we denote the set of all relations $\sigma \in v_{0}(X)$, for which in the block representation (7) of type $\left(p_{1}, p_{2}\right)$. 
1. The diagonal block $\sigma^{11}$ is the identity matrix,

2. In the block $\sigma^{12}$ consists of zeros,

3. The diagonal block $\sigma^{22}$ is a partial order (belongs to $v_{0}(M)$,

And let $W(p ; m)=\operatorname{card} w(p ; m)$. In addition, we assume:

$W(0 ; m)=T_{0}(m), m=0,1, \ldots, \quad W(p ; 0)=1, p=0,1, \ldots$

through $v(p, m)$ we denote the set of all relations $\sigma \in w(p, m) \quad$ Such that in the block representation (7) of type $\left(p_{1}, p_{2}\right)(=(p, m))$ the block $\sigma^{12}-$ nondegenerate. We use the notation $V(p ; m)=\operatorname{cardv}(p ; m)$ and we assume, by definition,

$V(0 ; m)=\delta_{0 m}, m=0,1, \ldots, \quad V(p ; 0)=1 p=0,1, \ldots$

For a relation $\sigma \in v_{0}(X)$ (in which, in contrast to the relations of $v_{0}^{0}(X)$, for all $x \in X$ justly $\sigma(x, x)=1$ ) support sets are the collections:

$S(\sigma)=\left\{y \in X: \sigma(x, y)=\delta_{x y}\right.$ for all $\left.x \in X\right\}$, $S^{\prime}(\sigma)=\left\{x \in X: \sigma(x, y)=\delta_{x y}\right.$ for all $\left.y \in X\right\}$. It is clear that the set $v(p, m)$ coincides with the set of all those relations $\sigma \in v_{0}(X)$, in which $S(\sigma)=\{1, \ldots, p\}$.
Theorem 6.1 For integer $p \geq 0$ and $m \geq 0$ the following equalities are hold:

$W(p ; m)=\sum_{r=0}^{m}\left(\begin{array}{l}m \\ r\end{array}\right) V(p+r ; m-r)$,

$V(p ; m)=\sum_{r=0}^{m}(-1)^{r}\left(\begin{array}{l}m \\ r\end{array}\right) W(p+r ; m-r)$.

Proof: Let $p \geq 1, m \geq 1$. It is easy to see that if $\sigma \in w(p ; m)$ then the set $P=\{1, \ldots, p\}$ is contained in the support set $S(\sigma)$. For any $\alpha \subseteq M, w_{\alpha}(p ; m)$ we denoted the set of all those relations $\quad \sigma \in w(p ; m), \quad$ which $S(\sigma)=P \bigcup \alpha$, thus breaking up the set of $w(p ; m)$ to pairwise disjoint classes $w_{\alpha}(p ; m)$.Therefore,

$W(p ; m)=\operatorname{card} w(p ; m)=\sum_{\alpha \subseteq M} \operatorname{card} w_{\alpha}(p ; m)$.

Obviously, when $\alpha=\varnothing$ the equalities $w_{\varnothing}(p ; m)=v(p ; m)$, and $w_{\varnothing}(p ; m)=V(p ; m) . \quad$ It is also easy to understand that if non-empty $\alpha, \beta \subseteq M$ such that $|\alpha|=|\beta|$, then:

$c a r d w_{\alpha}(p ; m)=c a r d w_{\beta}(p ; m)=\operatorname{card} w_{\gamma}(p ; m)$, where

$\gamma=\{p+1, \ldots, p+r\} \subseteq M, r=|\alpha|=|\beta| \in[1, m]$. 
Since for all $\sigma \in w_{\gamma}(p ; m)$ we have the equality $S(\sigma)=\{1, \ldots, p+r\}$, then :

$w_{\gamma}(p ; m)=v(p+r ; m-r)$, therefore:

$\operatorname{card}_{\gamma}(p ; m)=V(p+r ; m-r)$,

which proves (10). With the introduction of agreements (8) and (9) it is easy to verify that the equation (10) holds also for $p=0$ and $m=0$.

Let $\Delta$ we denoted the right-hand side

of the formula (11) then by the formula (10) we have the equality:

$\Delta=\sum_{r=0}^{m}(-1)^{r}\left(\begin{array}{l}m \\ r\end{array}\right) \sum_{s=0}^{m-r}\left(\begin{array}{l}m-r \\ s\end{array}\right) V(p+r+s ; m-r-s)$.

Replacing the index $S$ by $t=r+s$ and changing the order of summation, we obtain the following chain of equalities:

$$
\begin{aligned}
& \Delta=\sum_{t=0}^{m}\left[\sum_{r=0}^{t}(-1)^{r}\left(\begin{array}{l}
t \\
r
\end{array}\right)\right]\left(\begin{array}{l}
m \\
t
\end{array}\right) V(p+t ; m-t)= \\
& =\sum_{t=0}^{m} \delta_{t 0}\left(\begin{array}{l}
m \\
t
\end{array}\right) V(p+t ; m-t)=V(p ; m) .
\end{aligned}
$$

Theorem 6.2 For integer $p \geq 1$ and $m \geq 0$ the following equality are hold:

$$
V(p ; m)=\sum_{\substack{p_{1}+\ldots+p_{k}=p+m \\ p_{1} \geq p}}(-1)^{m+1-k} \frac{m !}{\left(p_{1}-p\right) ! p_{2} ! \ldots p_{k} !} W\left(p_{1}, \ldots, p_{k}\right) .
$$

Proof: Suppose that $m \geq 1$. By formula (11) the following equality holds:

$V(p ; m)=(-1)^{m}+\sum_{q=0}^{m-1}(-1)^{q}\left(\begin{array}{l}m \\ q\end{array}\right) W(p+q ; m-q)$.
In accordance with Lemma (3) in [7] for all $q=0,1, \ldots, m-1$ we have the equality:

$W(p+q ; m-q)=\sum_{q_{1}+\ldots+q_{r}=m-q}(-1)^{m-q-r} \frac{(m-q) !}{q_{1} ! \ldots q_{r} !} W\left(p+q, q_{1}, \ldots, q_{r}\right)$,

therefore

$$
\begin{aligned}
& V(p ; m)-(-1)^{m}=\sum_{q=0}^{m-1}(-1)^{q}\left(\begin{array}{l}
m \\
q
\end{array}\right) \sum(-1)^{m-q-r} \frac{(m-q) !}{q_{1} ! \ldots q_{r} !} W\left(p+q, q_{1}, \ldots, q_{r}\right)= \\
& =\sum_{\substack{q+q_{1}+\ldots+q_{r}=m \\
0 \leq q \leq m}}(-1)^{m-r} \frac{m !}{q ! q_{1} ! \ldots q_{r} !} W\left(p+q, q_{1}, \ldots, q_{r}\right) \text {. }
\end{aligned}
$$

Passed to the summation of all the variables simultaneously. Let $q_{0}=p+q$, then:

$$
V(p ; m)-(-1)^{m}=\sum_{\substack{q_{0}+q_{1}+\ldots+q_{r}=p+m \\ p \leq q_{0}<p+m}}(-1)^{m-r} \frac{m !}{\left(q_{0}-p\right) ! q_{1} ! \ldots q_{r} !} W\left(q_{0}, q_{1}, \ldots, q_{r}\right)
$$

The proof completes the change of variables

$$
\begin{gathered}
k=r+1, p_{i}=q_{i-1}, i=1, \ldots, k . \text { The case } \\
m=0 \text { is valid by virtue of the formula (9). }
\end{gathered}
$$

Remark 6.3: For any $n \in N$ and $p=1, \ldots, n$ through $A_{n}^{(p)}$ (through $\left.T_{0}^{(p)}(n)\right)$ denote the number of all labeled acyclic digraphs $\sigma \in A(X)$ (respectively labeled transitive digraphs $\left.\sigma \in v_{0}^{0}(X)\right)$ defined on the sets $X=\{1, \ldots, n\}$ and such that $S(\sigma)=\{1, \ldots, p\}$. It is clear that $T_{0}^{(p)}(n)=V(p ; n-p)$.

Thus, by virtue of Lemma (2), we can proof that the following theorem: 
Theorem 6.4 : For any $n \in N$ and $p=1, \ldots, n$ we have the following equality:

$$
\begin{aligned}
& T_{0}^{(p)}(n)=\sum_{\substack{p_{1}+\ldots+p_{k}=n \\
p_{1} \geq p}}(-1)^{n-p+1-k} \frac{(n-p) !}{\left(p_{1}-p\right) ! p_{2} ! \ldots p_{k} !} W\left(p_{1}, \ldots, p_{k}\right) \\
& A_{n}^{(p)}(n)=\sum_{\substack{p_{1}+\ldots+p_{k}=n \\
p_{1} \geq p}}(-1)^{n-p+1-k} \frac{(n-p) !}{\left(p_{1}-p\right) ! p_{2} ! \ldots p_{k} !} 2^{\left(n^{2}-p_{1}^{2}-\ldots p_{k}^{2}\right) / 2}
\end{aligned}
$$

The second formula is proved in Lemma 4 of (compare also with the expression (4).

\section{Reference}

[1] Al'Dzhabri Kh.Sh., Rodionov V.I. , The graph of partial orders, Vestn. Udmurt. Univ. Mat. Mekh. Komp'yut. Nauki, 2013, issue 4, pp. 3-12 (in Russian). DOI: 10.20537/vm130401

[2] Al'Dzhabri Kh.Sh. ,The graph of reflexivetransitive relations and the graph of finite topologies, Vestn. Udmurt. Univ. Mat. Mekh. Komp'yut. Nauki, 2015, Vol. 25, issue 1, pp. 3-11 (in Russian). \I DOI: $10.20537 / \mathrm{vm} 150101$

[3] Al' Dzhabri Kh.Sh., Rodionov V.I. ,The graph of acyclic digraphs, Vestn. Udmurt. Univ. Mat. Mekh. Komp'yut. Nauki, 2015, Vol. 25, issue 4, pp. 441-452 (in Russian). DOI: 10.20537/vm150401

[4] Comtet L., Recouvrements, bases de filtre et topologies d'un ensemble fini, C. R. Acad. Sci., 1966, Vol. 262, pp. A1091-A1094.es
[5] Erne M. , Struktur- und anzahlformeln fur topologien auf endlichen mengen, Manuscripta Math., 1974, Vol. 11, No. 3, pp. 221-259. DOI: 10.1007/BF01173716

[6] Borevich Z.I. "Enumeration of finite topologies", J. Sov. Math., 1982, Vol. 20, issue 6, pp. 2532-2545. II DOI: $10.1007 / \mathrm{BF} 01681470$

[7] Rodionov V.I. , On enumeration of posets defined on finite set, Siberian Electronic Mathematical Reports, 2016, Vol. 13, pp. 318-330 (in Russian). DOI: $10.17377 /$ semi.2016.13.026

[8] Evans J.W., Harary F., Lynn M.S. , On the computer enumeration of finite topologies, Comm. ACM, 1967, Vol. 10, issue 5, pp. 295-297. DOI: $10.1145 / 363282.363311$

[9] Gupta H. , Number of topologies on a finite set, Research Bulletin of the Panjab University (N.S.)\}, 1968, Vol. 19, pp. 231-241.

[10] Rodionov V.I. , On the number of labeled acyclic digraphs, Discrete Mathematics, 1992, Vol. 105, No. 1-3, pp. 319--321. DOI: 10.1016/0012365X(92)90155-9

[11] Rodionov V.I. , On recurrence relation in the problem of enumeration of finite posets, Siberian Electronic Mathematical Reports, 2017, Vol. 14, pp. 98-111 (in Russian). DOI: 10.17377/semi.2017.14.011

[12] Brinkmann G., McKay B.D. , Posets on up to 16 points, Order, 2002, Vol. 19, issue 2, pp. 147-179. ॥ DOI: 10.1023/A:1016543307592. 
حول اعداد البيانات المباشرة المتعدية والدائرية بوجود المجموعة الداعمة

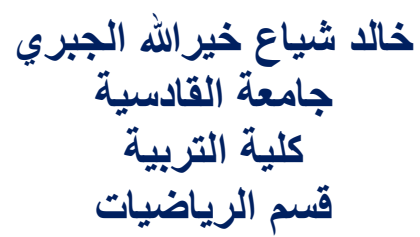

Khalid.aljabrimath@qu.edu.iq

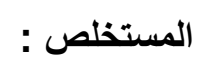

في الأعمال السابقة للمؤلف قدم مفهوم العلاقات الانعكاسية الثنائية الملاصقة من العلاقات الثنائية للمجموعة X و للنظام الجبري الذي يحوي كل العلاقات الثنائية للمجمو عة X وقدم جميع الأزواج غير مرتبة من العلاقات الثنائية الملاصقة ـ اذا كانت الــ X مجموعة منتهية بالتالي يمكن اعتبار هذا النظام الجبري هو

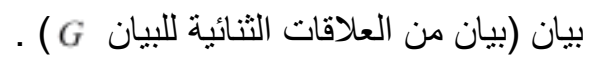
في البحث الحالي قدم مفهوم المجموعة الداعمة للبيانات المباشرة الدائرية و المتعدية حيث المجموعات

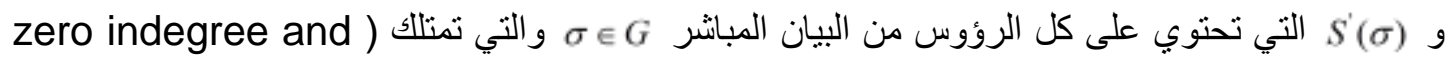
zero outdegree البيانات الدائرية والمتعدية المباثرة فأن التي تحسب كل هذه البيانات الدائرية و المتعدية المباثرة بوجود مفهوم المجمو عة الداعمة . الكلمات المفتاحية : تعداد الرسم البياني ، البيانات المباشرة الدائرية، البيانات المتعدية المباشرة. 